\title{
Muon detection in electron-positron annihilation for muon collider studies
}

\author{
N. Amapane ${ }^{\mathrm{a}, \mathrm{b}}$, M. Antonellic ${ }^{\mathrm{c}}, \mathrm{F}$. Anulli $^{\mathrm{d}}$, G. Ballerinie,, , L. Bandiera ${ }^{\mathrm{g}}, \mathrm{N}$. \\ Bartosik $^{\mathrm{b}}$, M. Bauce ${ }^{\mathrm{d}}$, A. Bertolin ${ }^{\mathrm{h}}$, C. Biino ${ }^{\mathrm{b}}$, O. R. Blanco-García ${ }^{\mathrm{c}}, \mathrm{M}$.

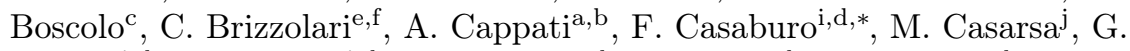

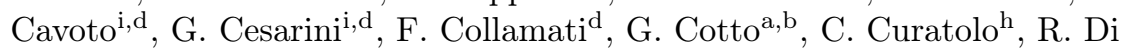

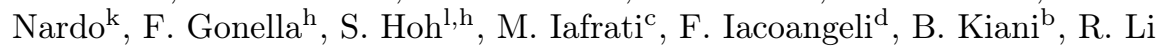

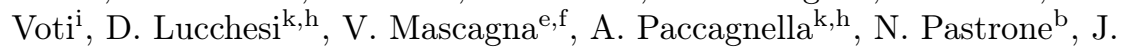

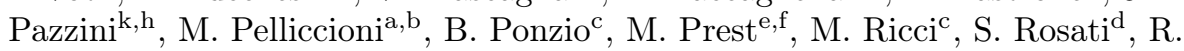 \\ Rossin $^{\text {l,h }}$, M. Rotondo ${ }^{\mathrm{c}}$, O. Sans Planell ${ }^{\mathrm{a}, \mathrm{b}}$, L. Sestini $^{\mathrm{h}}$, M. Soldani ${ }^{\mathrm{e}, \mathrm{f}}$, A.

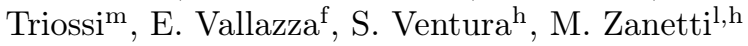 \\ ${ }^{a}$ Università degli Studi di Torino- Torino, Italy \\ ${ }^{b}$ INFN Sezione Torino- Torino, Italy \\ ${ }^{c}$ INFN Laboratori Nazionali di Frascati - Frascati, Italy \\ ${ }^{d}$ INFN Sezione Roma- Rome, Italy \\ ${ }^{e}$ Università degli Studi dell'Insubria - Como, Italy \\ ${ }^{f}$ INFN Sezione di Milano Bicocca - Milan, Italy \\ ${ }^{g}$ INFN Sezione di Ferrara - Ferrara, Italy \\ ${ }^{h}$ INFN Sezione di Padova - Padova, Italy \\ ${ }^{i}$ Sapienza Università di Roma- Roma, Italy \\ ${ }^{j}$ INFN Sezione di Trieste - Trieste, Italy \\ ${ }^{k}$ CERN - Geneva, Switzerland \\ ${ }^{l}$ Università di Padova - Padova, Italy \\ ${ }^{m}$ Institut Pluridisciplinaire Hubert Curien, Strasbourg, France
}

\begin{abstract}
The investigation of the energy frontier in physics requires novel concepts for future colliders. The idea of a muon collider is very appealing since it would allow to study particle collisions at up to tens of $\mathrm{TeV}$ energy, while offering a cleaner experimental environment with respect to hadronic colliders. One key element in the muon collider design is the low-emittance muon production. Recently, the Low EMittance Muon Accelerator (LEMMA) collaboration has explored the muon pair production close to its kinematic threshold by annihilating $45 \mathrm{GeV}$ positrons with electrons in a low $Z$ material target. In this configuration, muons
\end{abstract}

\footnotetext{
* Corresponding author

Email address: fausto.casaburo@uniroma1.it (F. Casaburo)
} 
are emerging from the target with a naturally low-emittance. In this paper we describe the performance of a system, to study this production mechanism, that consists in several segmented absorbers with alternating active layers composed of fast Cherenkov detectors together with a muon identification technique based on this detector. Passive layers were made of tungsten. We collected data corresponding to muon and electron beams produced at the $\mathrm{H} 2$ line in the North Area of the European Organization for Nuclear Research (CERN) in September 2018.

Keywords: Muon Collider, Cherenkov detectors

\section{Contents}

Introduction

1 Experimental Setup 4

2 Absorbers system performance studies 99

2.1 Data acquisition . . . . . . . . . . . . . . . . . 9

2.2 Tracker - absorbers correlation . . . . . . . . . . . 10

2.3 Lead Glass calibration . . . . . . . . . . . . . . . . . . . 12

2.4 HOrizontal Smart Absorber efficiency _. . . . . . . . . . . 13

2.5 HORSA electron misidentification . . . . . . . . . . . 13

2.6 HORSA muon detection . . . . . . . . . . . . . . . . . . 14

3 Particles detection in positron beam data with the target 15

3.1 Data to Monte Carlo simulations comparisons . . . . . . . . . . 18

4 Conclusions $\quad 19$

$\begin{array}{ll}\text { Acknowledgements } & 20\end{array}$ 


\section{Introduction}

Exploring the high energy frontier represents a great opportunity to investigate the fundamental laws of nature. This requires a particle collider able to accelerate elementary particles to the highest possible energy. A muon collider represents an appealing though challenging solution for the aforementioned purpose $[1,2]$. Previous studies claimed that a muon collider is conceivable to reach the multi-TeV energy frontier with the possibility to study Higgs boson properties [2]. Muons in a circular storage ring are in fact emitting much less synchrotron radiation than electrons with the same energy; therefore, muons accelerated in a circular collider can reach a center-of-mass (CM) energy higher than electrons in the same ring. However, the muon lifetime of approximately $2 \mu \mathrm{s}$ is a limiting factor on the design of a muon accelerator complex, requiring a fast accelerating complex. Moreover, reaching a small emittance is one of the crucial aspects to achieve a high luminosity in a muon collider [3].

The LEMMA project [4] aims to study the possibility of producing muons from the $e^{+} e^{-}$annihilation process. A high intensity positron beam, with energy just above the $43.7 \mathrm{GeV}$ threshold for muon pair production through the $e^{+} e^{-} \rightarrow$ $\mu^{+} \mu^{-}$process, impinging on a low $Z$ fixed target [5] could produce muons with naturally small divergence, resulting in a low transverse emittance. This would avoid the need of a subsequent beam cooling stage, otherwise required in a muon production scheme based on pion decays as considered in the Muon Accelerator Program (MAP) [6]. Experimental data in the energy regime close to muon pair production threshold are not frequent since most of the measurements of the $e^{+} e^{-} \rightarrow \mu^{+} \mu^{-}$process are performed at higher CM energy values [7]. It is therefore necessary to measure the aforementioned production cross section and the produced muon pair kinematic properties for several values of the CM energy near the muon pair production threshold to probe the validity of the predictions.

While the leading-order Quantum ElectroDynamics (QED) $e^{+} e^{-} \rightarrow \mu^{+} \mu^{-}$ cross section calculation is well established, higher order radiative effects, due 
to Coulomb interaction, might gain importance close to the kinematic threshold when evaluating both the muon pair production rate and their angular distribution [8]. A dedicated experimental setup has been arranged to study the muon pair production through $45 \mathrm{GeV}$ positrons impinging on a Beryllium or Carbon target in different data-taking campaigns [9].

The aim of this paper is to describe a system made of two segmented and instrumented absorbers, that has been used to effectively study the $e^{+} e^{-} \rightarrow$ $\mu^{+} \mu^{-}$process near the muon pair production threshold region.

These absorbers were part of the apparatus installed at the CERN North Area beam lines during the 2017 and 2018 data-taking periods. These were deployed to identify both positive and negative muons and discriminate them from electrons and positrons. The absorbers were initially conceived as massive but portable devices that could be used as sampling calorimeters to study the development of hadronic showers initiated by the Large Hadron Collider (LHC) beam interactions [10]. Their performances for muon identification have been tested using both electron and muon beams. Results regarding the performances of one of these absorbers, named HOrizontal Smart Absorber (HORSA) in the following, are described in Section 2. To validate these performances, we measured the ratio of the number of muons to the number of electrons in events produced from a $45 \mathrm{GeV}$ positron beam data hitting a Carbon target and compared with a Monte Carlo (MC) simulation, as described in Section 3. Lastly, a discussion of the obtained results is reported in the conclusions (Section 4).

\section{Experimental Setup}

The experimental setup arranged to collect data consists of an approximately $23 \mathrm{~m}$ long apparatus, shown in Fig. 1 (vacuum pipe, target and magnetic spectrometer region) and Fig. 2 (absorbers region) that was installed in the H2 beam line of the CERN North Area during a data-taking campaign in summer 2018 to measure with high precision trajectories and momenta of the two final state muons as well as the direction of the incoming positrons. As shown in Fig. 
1 , the coordinate system has the $z$-axis along the beam, the $x$-axis pointing upwards and $y$-axis pointing towards the reader. In both Figures 1 and 2, lengths are expressed in $\mathrm{cm}$.

Upstream of the fixed target (Fig. 1), positrons from the beam are crossing a pair of $2 \times 2 \mathrm{~cm}^{2}$ silicon sensors, constituted by two layers of orthogonal microstrips in order to measure the incoming particle direction and position. A scintillator placed upstream of the target was used for real-time event selection and data-acquisition purposes (trigger).

Another silicon sensor pair is placed downstream of the target (Fig. 1) in order to measure the direction of emerging particles before these enter a dipole magnet with a $2.01 \mathrm{~T}$ field along $y$, extended in a region of approximately $\pm 100 \mathrm{~cm}$ along $z$.

Each secondary particle (being it $e^{ \pm}$or $\mu^{ \pm}$) is individually reconstructed and its momentum is determined by measuring its deflection in the $x$ - $z$ plane with a two arms silicon sensor spectrometer located downstream of the magnet (Fig. 1).

After being tracked by the aforementioned spectrometer, particles are entering the absorbers region (Fig. 2) where the detectors are arranged along two arms, following the expected trajectory of positive and negative particles. Each arm is constituted by two scintillators serving as a trigger and the massive absorbers. An iron block placed across both arms is employed to shield Drift Tube (DT) muon chambers located further downstream (the DT are not displayed in Fig. 2 and they have not been used in this measurement).

Each active absorber is made of a three unit Lead Glass (LG) electromagnetic calorimeter, each unit featuring a $40 \mathrm{~cm}$ deep truncated pyramid shape. The LG photomultiplier (PMT) signals were processed by a 12 bit digitizer [11]. The instrumented absorber is installed immediately after the LG calorimeter.

The absorber named HORSA, the only device for which performances are presented in this paper, is installed in the spectrometer arm where the negative particles (either $e^{-}$or $\mu^{-}$) are deflected by the magnetic field. It is equipped with 1 inch thick fused silica layers where secondary charged particles are pro- 


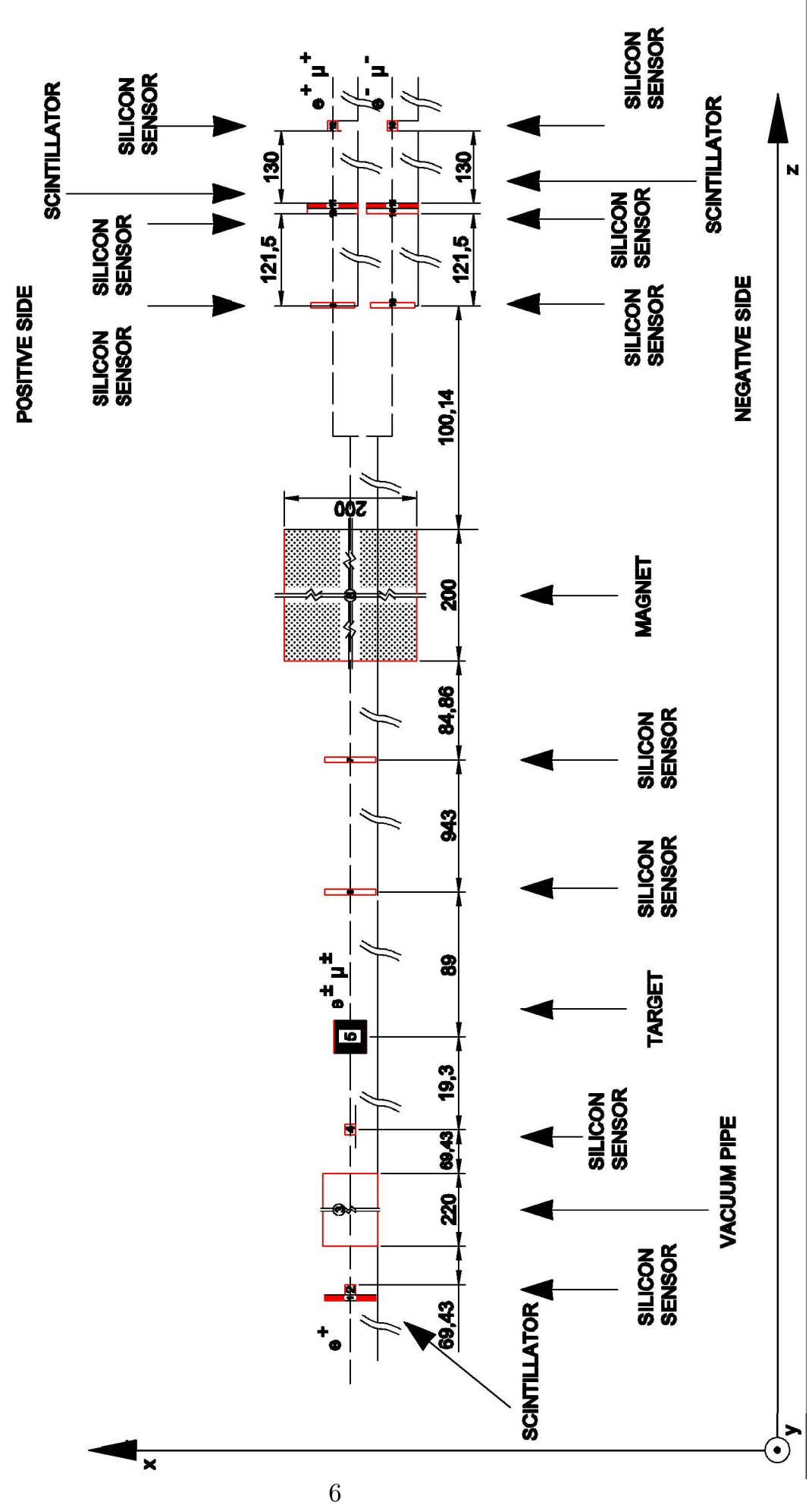

Figure 1: Magnetic spectrometer setup installed at the H2 beam line of CERN to study the particles emerging from a low $Z$ target in positron annihilation processes. Lengths are expressed in $\mathrm{cm}$. 
ducing Cherenkov light. Three PMTs are used to detect the light produced in each of the fused silica layers. The light is transmitted to the PMTs by internal reflection along the $x$ direction. HORSA PMTs were processed by a 14 bit digitizer [12]. A sequence of alternating active and passive layers along $z$ are used to filter muons against electrons. Among the first (second) pair of fused silica layers a $5 \mathrm{~cm}(3 \mathrm{~cm})$ tungsten shield is inserted. Among the two fused silica layer pairs a passive element made of a $23 \mathrm{~cm}$ thick tungsten layer is inserted.

Two trigger configurations were used for the shared silicon sensors and calorimeters Data Acquisition (DAQ). A first one, named single, was based on the signal produced by the scintillator placed upstream of the target (Fig. 1). A second one, named muon, was based on the coincidence of the same upstream scintillator signal with the signals of two pairs of additional scintillators placed upstream of the LG blocks and downstream of the DT chambers respectively (Fig. 2), on each of the two arms of the detector (i.e. the absorber region showed in Fig. 2). The single trigger was used to select and record events with no bias on the final state while the muon trigger was used to enhance the content of $\mu^{+} \mu^{-}$events in the recorded data sample.

Two kinds of DAQ system have been used to record event information from the different subdetectors. The DAQ system for all subdetectors, except the DT chambers, is based on an external trigger signal given by a combination of the scintillator fast signals (the aforementioned single and muon combinations). The DT chambers used instead a fast, $40 \mathrm{MHz}$, trigger-less, DAQ system, developed for the Run 3 data taking period of the Compact Muon Solenoid (CMS) detector [13]. The trigger signal from the scintillators is also shared to the DT DAQ system for subdetector off-line synchronisation and complete event building. 


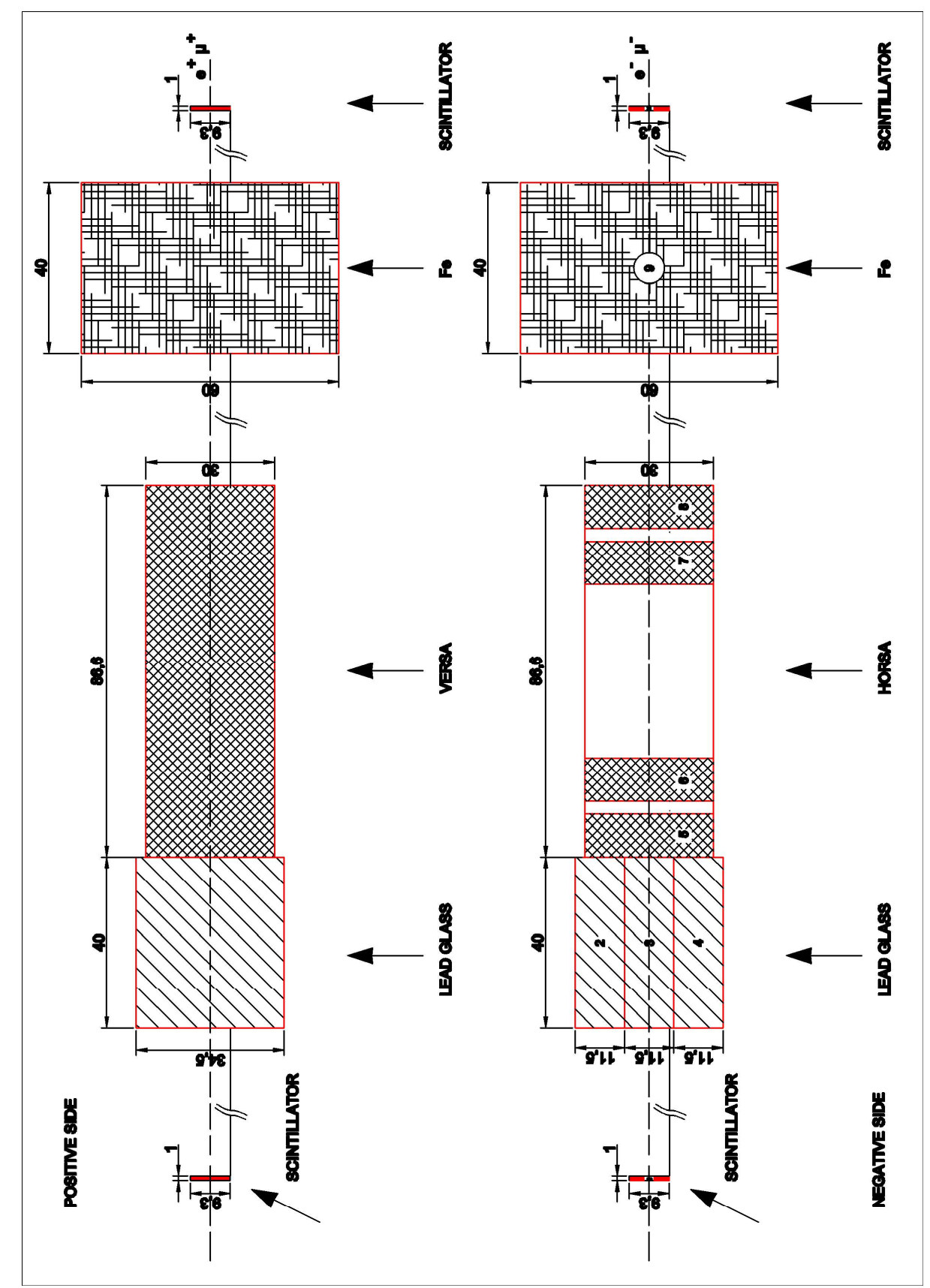

Figure 2: Absorbers region located downstream the magnetic spectrometer. Lengths are expressed in $\mathrm{cm}$. 


\section{Absorbers system performance studies}

\subsection{Data acquisition}

Data from events generated at the $\mathrm{H} 2$ beam line were recorded during the summer 2018 data-taking campaign. Electron and muon beams of different energies without the target in position were delivered to the experimental region, as well as a $45 \mathrm{GeV}$ positron beam hitting the target in its position. Muon beams with $E_{0}=22 \mathrm{GeV}$ energy, without any target in place, and with both magnetic field directions, have been used for the alignment of the silicon detectors and the DT chambers.

Muon and electron beams, both with the same energy $E_{0}$ and no target inserted are also used to estimate the detector's performances. This energy is approximately equal to the mean energy of the muons produced in the $e^{+} e^{-} \rightarrow \mu^{+} \mu^{-}$process when initiated by a $45 \mathrm{GeV}$ positron beam. The magnet is operated to deflect particles with energy $E_{0}$ into the downstream double-arm section: particles with this energy are in fact hitting the central unit of each LG calorimeter, depending on the sign of their charge. In addition to the aforementioned calibration runs, different data-taking runs were carried out with a $45 \mathrm{GeV}$ positron beam delivered to different kinds of targets to study muon production close to the kinematic threshold of the $e^{+} e^{-} \rightarrow \mu^{+} \mu^{-}$process. The results presented in this paper are based on the data collected during runs in which two Carbon targets with $2 \mathrm{~cm}$ and $6 \mathrm{~cm}$ thickness and a diameter of $4 \mathrm{~cm}$ were present (Sec. 3). The positron beam had a pulsed shape with 4 spills per minute, each spill lasting $4.8 \mathrm{~s}$ with a typical intensity of $5 \cdot 10^{6}$ positrons per spill. The spot size was $\sim 2 \times 2 \mathrm{~cm}^{2}$ with an angular spread of $\sim 300 \mu \mathrm{rad}$. With the chosen collimator settings the momentum spread was below $1.5 \%$ [14] and the purity of the beam was in the range $95-99 \%$ [15].

During the data-taking, information about each particle arrival time, released energy in each of the absorbers and positions detected by the silicon trackers is recorded. For the runs with the $45 \mathrm{GeV}$ positron beam hitting the target, muon and single triggers were included to start the DAQ system with a 
prescaling factor of about $3 \cdot 10^{4}$.

Events with $\mu^{+} \mu^{-}$or $e^{+} e^{-}$pairs in the final state were identified through the LG and the HORSA absorber located on the negative side of the spectrometer, i.e. based only on the identification of the negative particle in the final state. Events with a $\mu^{+} \mu^{-}$pair in the final state are mainly due to the $e^{+} e^{-}$ annihilation process in the target. Events with a $e^{+} e^{-}$pair in the final state are produced either in $e^{+} e^{-}$Bhabha scattering of the impinging positron beam with an atomic electron in the target material or in pair production from a high energy photon in the target [16].

Electrons reaching the absorbers region are producing an electromagnetic shower almost entirely contained in the LG central unit while negative muons are releasing a small amount of their energy in the detector materials hence crossing entirely the LG and HORSA detectors. Muons are expected to produce a signal both in the LG central unit and in all the HORSA active layers. Only a fraction of the energy lost by each muon is deposited as Cherenkov light and eventually detected by the PMTs. A muon event candidate is defined based on the time coincidence of signals from the PMTs corresponding to all the five trigger scintillators, the central LG unit and the four HORSA active layers, with an anti-coincidence requirement with respect to the two external LG units.

Exploiting the different behaviour of muons and electrons in the apparatus, the ratio of the number of events with a muon to those with an electron, $N_{\mu^{-}} / N_{e^{-}}$, reaching the absorber region can be computed and eventually be compared with a $\mathrm{MC}$ simulation of the experimental setup.

\subsection{Tracker - absorbers correlation}

As mentioned before, the experimental apparatus is arranged so that the magnet deflects particles with energy $E_{0}$ towards the central unit of the LG (block 3 in Fig. 2). As a first step, this analysis studied the relation between the particle's $x$ position measured by the tracker (Fig. 1) positioned before

the first scintillator of the negative arm and the energy released $E_{3}$ in the central LG unit (subscript number refers to Fig. 2). Fig. 3 shows the average 
electron (muon) released energy $\left\langle E_{3}\right\rangle$ in the central LG unit in blue (green), as a function of the $x$ position measured by the tracker. The $x$-scale refers to the local coordinate system of the tracker. Particles in the range $6 \mathrm{~cm}<x<14 \mathrm{~cm}$ are the ones that traverse the whole central LG unit. The noticeable negative correlation is due to the finite energy spread of the beam. Particles crossing the tracker at lower $x$ correspond to those closer to the beam line, less deflected because of their higher momentum.

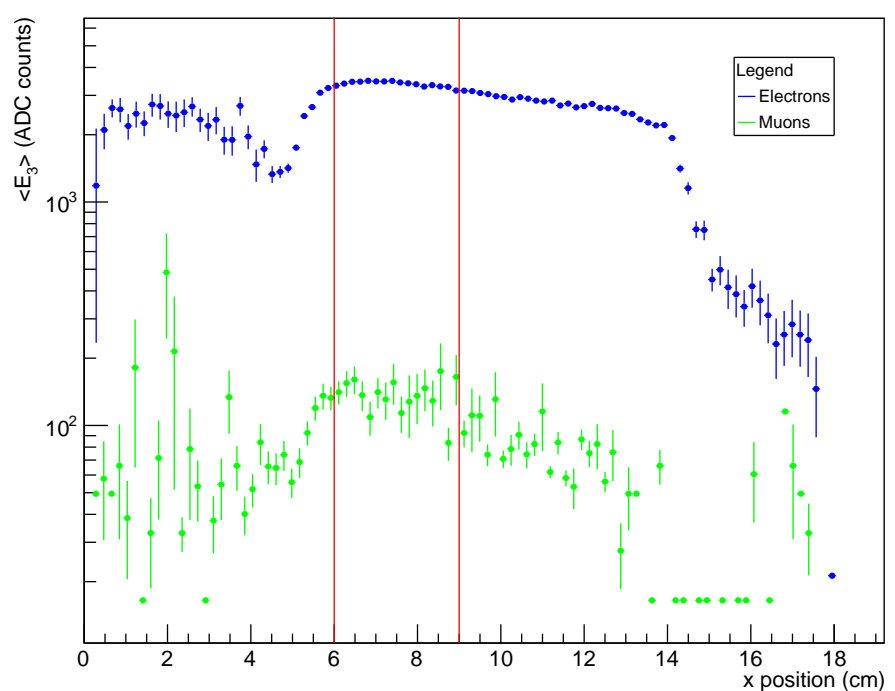

Figure 3: Average energy released $\left\langle E_{3}\right\rangle$ by $22 \mathrm{GeV}$ electrons (blue, on the top) and muons (green, on the bottom) in the central LG unit as a function of the local $x$ position measured by the tracker. Vertical solid lines are used to mark the region $6 \mathrm{~cm}<x<9 \mathrm{~cm}$, considered for further analysis.

The largest released energy occurs for particles crossing the tracker with coordinates in the range $6 \mathrm{~cm}<x<9 \mathrm{~cm}$, both for muons and electrons. This requirement is applied to define samples of electrons or muons considered for the studies described in the following. 


\subsection{Lead Glass calibration}

Data collected during a run with an electron beam entering the apparatus and no target in place has been considered to study the released energy in the central unit of the LG $E_{3}$. This is needed to calibrate the energy response of this detector. Events with a time coincidence between signal in the scintillator (Fig. 2) and in the three LG units were selected. The spectrum of the energy released in the central LG unit by the selected electron candidates is shown in Fig. 4 together with a Gaussian function interpolating the spectrum.

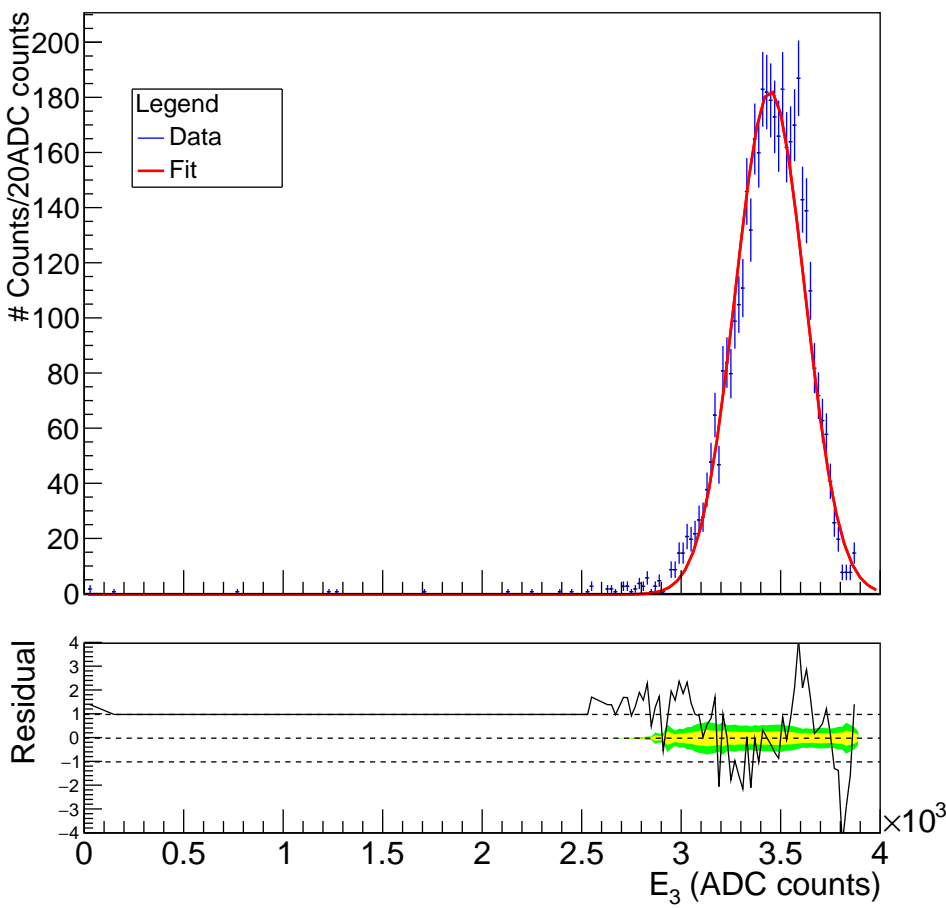

Figure 4: Energy released $E_{3}$ by electron candidates in the central LG in a $22 \mathrm{GeV}$ electron beam run with no target in place. The distribution is interpolated with a Gaussian function (solid line) of which the lower panel of the plot shows the residual. This procedure serves as calibration of the LG.

The Gaussian interpolation results in a mean value of $\mu=3447 \mathrm{ADC}$, the square root of the variance being $\sigma=174 \mathrm{ADC}$, with a reduced chi-squared 
(least squares) of $\chi^{2} / \nu=175 / 70$, where $\nu=70$ is the number of the degrees of freedom. Electrons are expected to release all of their energy in the LG volume hence it is possible to calibrate the LG energy response according to the relation $1 \mathrm{GeV}=(156.7 \pm 7.9) \mathrm{ADC}$ counts, assuming a linear response. The quoted uncertainty is the statistical uncertainty, given that the systematic uncertainties on this relation are negligible compared to the statistical one on the studies presented in the following.

\subsection{HOrizontal Smart Absorber efficiency}

The LG calibration allows the identification of muon candidates and their discrimination from other secondary particles based on the energy they release in the central LG unit, $E_{3}$. Muon candidates are required to have $E_{3}<1 \mathrm{GeV}$ (this value has been estimated by MC simulations).

The efficiency of each HORSA active layer has been studied using a muon beam with energy $E_{0}$. The efficiency of each HORSA active layer $j=5,6,7,8$ (numbers refer to Fig. 2) is defined as:

$$
\varepsilon_{j}=\frac{N_{4}}{N_{3}(j)}
$$

where $N_{4}$ represents the number of events with signals in time coincidence in all four layers while $N_{3}(j)$ represents the number of events with signals in time coincidence in all the layers but the $j$ one. The overall efficiency of the HORSA absorber is defined as

$$
\varepsilon_{\mathrm{HORSA}}=\prod_{j=5}^{8} \varepsilon_{j}
$$

Results obtained from the run with a muon beam of energy $E_{0}$ and no target in place are given in Tab. 1, together with the corresponding statistical uncertainties evaluated assuming binomial statistics.

\subsection{HORSA electron misidentification}

To evaluate the probability to mistake electrons (produced by positrons hitting a target) for muons (also produced by positrons on target), the electron misidentification in the HORSA absorber is evaluated using data collected in a 
Table 1: Efficiencies to detect a $22 \mathrm{GeV}$ muon in each of the fused silica HORSA layers together with the overall HORSA efficiency, as defined in Equations 1 and 2 respectively.

\begin{tabular}{lll}
\hline$j$ & $\varepsilon_{j}(\%)$ & $\varepsilon_{\text {HORSA }}(\%)$ \\
\hline 5 & $97.04 \pm 0.56$ & \\
6 & $95.50 \pm 0.66$ & \\
7 & $93.26 \pm 0.98$ & $84.2 \pm 1.4$ \\
8 & $97.44 \pm 0.79$ & \\
\hline
\end{tabular}

run with an electron beam with energy $E_{0}$ crossing the apparatus. The electron misidentification in the HORSA absorber is given by:

$$
e_{\text {misID }}^{-}=\frac{N_{\mathrm{HORSA}}}{N_{\text {Tot }}}
$$

where $N_{\text {HORSA }}$ is the number of events with signals in time coincidence in the four HORSA layers and $N_{\text {Tot }}$ is the total number of recorded events.

In the considered run of data-taking $N_{\mathrm{HORSA}}=1$ and $N_{\text {Tot }}=21504 \mathrm{so}$, exploiting the Feldman-Cousins (FC) approach for confidence interval definition, an Upper Limit (UL) on $e_{\text {misID }}^{-}$at $2 \cdot 10^{-4}$ has been set at $90 \% C L$. Thanks to a $\mathrm{MC}$ simulation of electron beam crossing the apparatus we estimate that the observed electron contamination is due to the not fully contained electromagnetic shower leaking outside of the LG volume.

\subsection{HORSA muon detection}

While crossing the apparatus, muons are expected to produce a signal in the central LG unit and in all the four HORSA active layers.

The observable energy released by muons in the apparatus is therefore:

$$
E_{\text {obs }}=E_{3}+E_{\mathrm{HORSA}} .
$$

where $E_{\mathrm{HORSA}}=\sum_{j=5}^{8} E_{j}$ (subscript numbers refer to Fig. 2) is the released energy in the different HORSA layers and $E_{3}$ has been already defined and is shown in Fig. 5. 


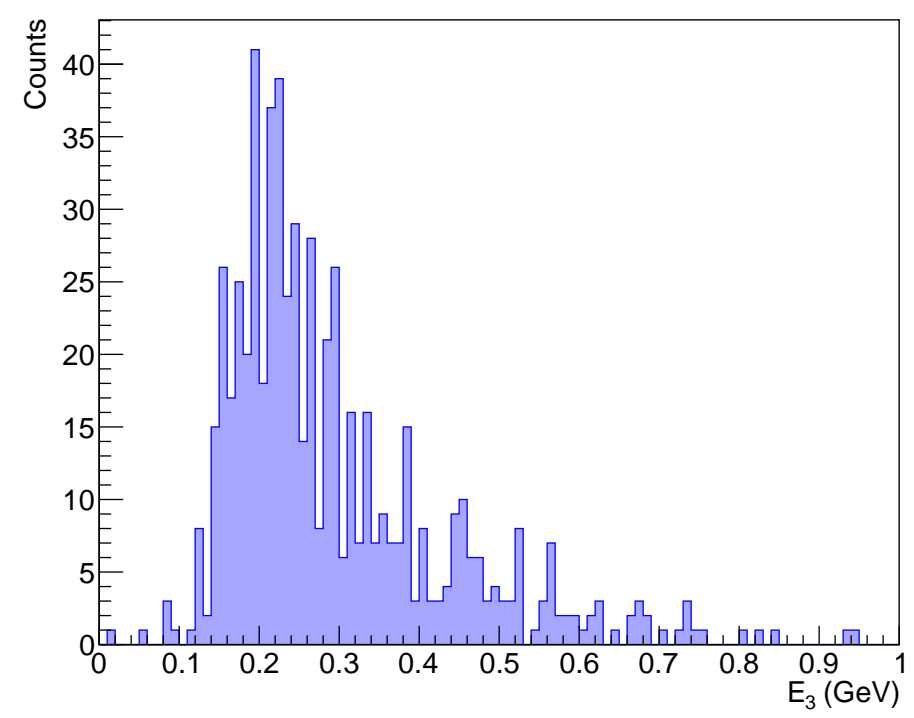

Figure 5: Energy released in the central LG unit $E_{3}$, for events recorded during a $22 \mathrm{GeV}$ muon beam data-taking run.

The correlation between $E_{3}$ and $E_{H O R S A}$, shown in Fig. 6, illustrates that the total energy released by muons with energy $E_{0}$ in the HORSA detector is $E_{\text {HORSA }}<2000$ ADC most of the time. This requirement can be therefore used to define a region for muon identification in HORSA detector. This criteria will be used, together with requirements on the energy deposited in the LG detectors, to identify muons produced in the events recorded during the $45 \mathrm{GeV}$ $e^{+}$beam run hitting the target.

\section{Particles detection in positron beam data with the target}

The calibrated detector has been exploited to measure the $N_{\mu^{-}} / N_{e^{-}}$events ratio, estimating the yields of $\mu^{-}\left(N_{\mu^{-}}\right)$and of $e^{-}\left(N_{e^{-}}\right)$, both with energy $E_{0}$. The yields are evaluated analyzing events collected during runs in which a positron beam is impinging on Carbon targets with different thicknesses, $2 \mathrm{~cm}$ and $6 \mathrm{~cm}$ respectively. 


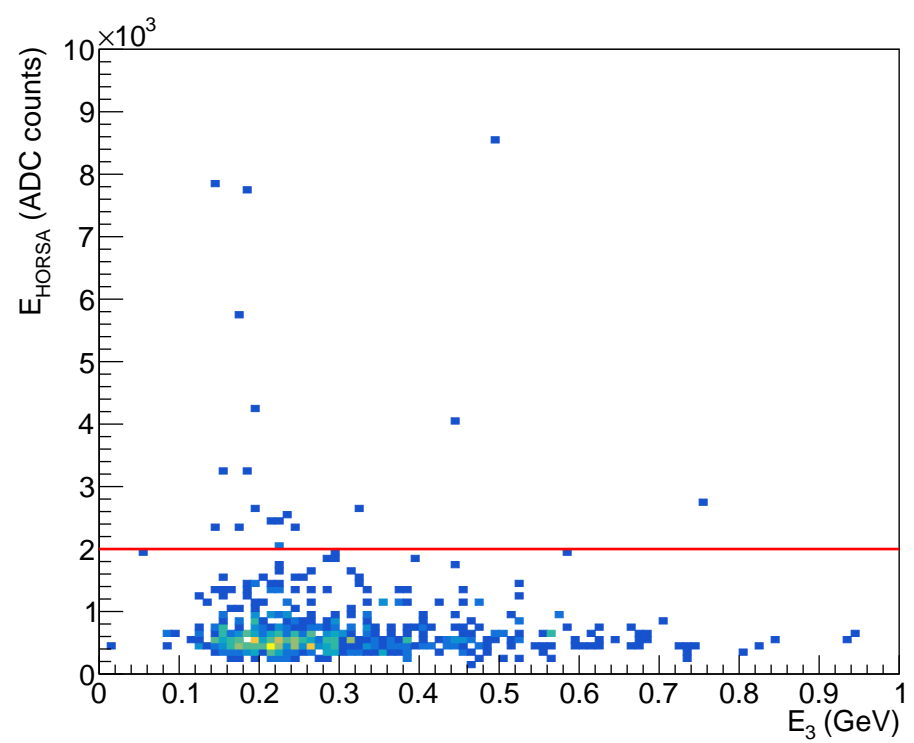

Figure 6: Energy released in HORSA $E_{\text {HORSA }}$ as a function of released energy in the central LG unit $E_{3}$ for events recorded during a $22 \mathrm{GeV}$ muon beam data-taking run. The solid line represents the requirement applied in the signal region definition. 
Considering the setup and numbering scheme shown in Fig. 2, candidate muon events have been selected requiring the time coincidence of signals in the two trigger scintillators (the first one upstream of the LG block and the second one downstream of the muon chamber), the LG central unit and all the HORSA layers; anti-coincidence with the LG external units (numbered 2 and 4 in Fig. 2 ) is also required.

The contribution due to fake signal events representing a background contribution in the muon identification region, defined requiring $E_{\mathrm{HORSA}}<2000 \mathrm{ADC}$ is evaluated using the sideband-substraction method. An orthogonal sideband region is defined by requiring $E_{\mathrm{HORSA}} \geq 2000 \mathrm{ADC}$. Assuming a uniform background distribution across the considered $E_{\text {HORSA }}$ range, the signal yields after the sideband-substraction are given by:

$$
N_{\mu_{\text {det }}^{-}}=N_{\mu^{-}}-\alpha N_{\text {side }}
$$

where $N_{\mu^{-}}$and $N_{\text {side }}$ are the number of candidate muon events in the signal region and the number of background events in the sideband region, respectively. The parameter $\alpha$ takes into account the different width of the signal and sideband region; this has been fixed to

$$
\alpha=\frac{2000 \mathrm{ADC}}{E_{\max }-2000 \mathrm{ADC}}
$$

where $E_{\max }$ is the upper edge of the sideband region, being approximately $30000 \mathrm{ADC}$ in the two considered runs.

Considering the setup and numbering scheme shown in Fig. 2, an electron from the $e^{+} e^{-}$final state is selected requiring the time coincidence of signals in the two trigger scintillators (one for each arm of the detector) positioned upstream of the LG blocks and the central unit of the LG in the negative arm. Time anti-coincidence with the signal from the two trigger scintillators (one for each arm of the detector) positioned downstream of the muon chambers is also required.

Defining as $N_{e_{d e t}^{-}}$and $\varepsilon_{e^{-}}$the number of detected electrons and the efficiency to detect them, respectively, and taking into account the evaluated muon de- 
tection efficiency $\epsilon_{H O R S A}$, the ratio between the produced muons and electrons is:

$$
\frac{N_{\mu^{-}}}{N_{e^{-}}}=\frac{N_{\mu_{\text {det }}^{-}}}{\varepsilon_{\mathrm{HORSA}}} \cdot \frac{\varepsilon_{e^{-}}}{N_{e_{\text {det }}^{-}}}
$$

The efficiency to detect an electron has been estimated being close to unity through a MC simulation. Therefore, the overall efficiency ratio can be assumed to be $\varepsilon \sim \varepsilon_{\mathrm{HORSA}}$ and $N_{e_{d e t}^{-}} \sim N_{e^{-}}$. Under these assumptions Eq. 7 can be reduced to:

$$
\frac{N_{\mu^{-}}}{N_{e^{-}}}=\frac{1}{\varepsilon_{\mathrm{HORSA}}} \cdot \frac{N_{\mu_{\text {det }}^{-}}}{N_{e^{-}}} .
$$

The measured $N_{\mu^{-}} / N_{e^{-}}$ratios for runs corresponding to Carbon targets of different thicknesses exposed to the positron beam are summarized in Tab. 2 . The uncertainties on the ratios have been evaluated propagating statistical uncertainties on $N_{\mu_{d e t}^{-}}$and $N_{e^{-}}$, assuming a Gaussian uncertainty when counts were $>30$ and a Poisson uncertainty otherwise. Several source of systematic uncertainties have been taken into account. The majority of the systematic effects are expected to affect the electron and muon detection in a similar way, hence will cancel in the ratio at first order. The limited amount of recorded muon events prevented from further investigations on additional systematic effects.

Table 2: Observed electron and muon yields together with the corresponding ratio $N_{\mu^{-}} / N_{e^{-}}$ for two runs corresponding to different Carbon target thicknesses.

\begin{tabular}{llll}
\hline Thickness $(\mathrm{cm})$ & $N_{e^{-}}$ & $N_{\mu_{\text {det }}^{-}}$ & $N_{\mu^{-}} / N_{e^{-}}$ \\
\hline 2 & $85.0 \pm 9.2$ & $37.0 \pm 6.6$ & $(5.1 \pm 1.1) \cdot 10^{-1}$ \\
6 & $78.0 \pm 8.8$ & $5.0_{-3.0}^{+4.1}$ & $\left(7.6_{-4.6}^{+6.4}\right) \cdot 10^{-2}$ \\
\hline
\end{tabular}

\subsection{Data to Monte Carlo simulations comparisons}

The $e^{+} e^{-} \rightarrow \mu^{+} \mu^{-}$cross section is known to be significantly smaller than the $e^{+} e^{-} \rightarrow e^{+} e^{-}$one, with a ratio of approximately $\frac{1}{8}$ for CM energies close to 
the kinematic threshold for muon pair production. This value has been obtained from the BabaYaga MC generator [17] using positrons and free electrons at rest in the initial state.

In order to validate the absorber system particle detection ability leading to the results shown in Tab. 2, a GEANT4 $[18,19]$ based MC simulation of particle electromagnetic interactions with matter has been developed, to compare the $N_{\mu^{-}} / N_{e^{-}}$events ratio obtained in data with theoretical predictions.

Positrons beams along the $z$-axis, made of $10^{10} 45 \mathrm{GeV}$ primary $e^{+}$with characteristics equivalent to the experimental ones and hitting Carbon targets (both with $2 \mathrm{~cm}$ and $6 \mathrm{~cm}$ thickness) have been simulated in order to evaluate the expected number $N_{e_{M C}^{-}}$of produced $e^{-}$and the $N_{\mu_{M C}^{-}}$of produced $\mu^{-}$.

The electron and muon yields predicted by the simulations, as well as their ratio are reported in Tab. 3 for the two target thicknesses considered.

Table 3: Predicted electron and muon yields together with the corresponding ratio $N_{\mu^{-}} / N_{e^{-}}$ for two simulated runs corresponding to different Carbon target thicknesses.

\begin{tabular}{llll}
\hline Thickness $(\mathrm{cm})$ & $N_{e_{M C}^{-}}$ & $N_{\mu_{M C}^{-}}$ & $N_{\mu_{M C}^{-}} / N_{e_{M C}^{-}}$ \\
\hline 2 & $2400 \pm 49$ & $1481 \pm 38$ & $(6.2 \pm 1.3) \cdot 10^{-1}$ \\
6 & $(521 \pm 2.3) \cdot 10^{2}$ & $2870 \pm 54$ & $(5.51 \pm 0.26) \cdot 10^{-2}$ \\
\hline
\end{tabular}

The experimental results reported in Tab. 2 are well in agreement, within uncertainties, with the corresponding predictions from $\mathrm{MC}$ simulations, reported in Tab. 3.

\section{Conclusions}

The implementation of the LEMMA muon collider concept needs an intense positron beam to produce muons at a significant rate. The knowledge of the muon production cross section close to its kinematic threshold is extremely important for a muon collider design based on this concept. 
In this paper we reported the results of a muon identification technique based on segmented massive absorbers that can be used in experiments to measure the properties of the muons produced by the $e^{+} e^{-}$annihilation at kinematic threshold of muon pair production. Detected events allowed testing the performance of the considered absorbers system. A comparison with a MC simulation resulted in a good agreement between experimental observations and predictions. This apparatus and the described particle identification method can be further deployed for future studies of the $e^{+} e^{-} \rightarrow \mu^{+} \mu^{-}$process at its kinematic threshold energy.

In order to increase statistics and reduce backgrounds, i.e. to provide a meticulous measurement of $e^{+} e^{-} \rightarrow \mu^{+} \mu^{-}$cross section, more accurate tracking devices, alignment infrastructures and a more efficient trigger and readout systems will be needed. An upgrade of the LEMMA experimental layout is foreseen in order to accumulate more data at the muon pair production threshold energy.

With an adequately low energy spread and with a larger beam rate this apparatus might also be employed to search for bound states as the true muonium $[20]$.

\section{Acknowledgements}

We would like to warmly thank the Super Proton Synchrotron (SPS) staff and the Large Scale Metrology (LSM) group, in particular Henrik Wilkens and Nikolaos Charitonidis, for their support during installation and data taking. This work has been supported by the ERC CoG GA 615089 CRYSBEAM.

\section{References}

[1] R. Franceschini, M. Greco, Higgs and bsm physics at the future muon collider (2021). arXiv:2104.05770.

[2] The Muon Accelerator Program, https://map.fnal.gov/. 
[3] K. Long, D. Lucchesi, M. Palmer, N. Pastrone, D. Schulte, V. Shiltsev, Muon colliders to expand frontiers of particle physics, Nature Physics 17 (2021) 1-4. doi:10.1038/s41567-020-01130-x.

[4] M. Antonelli, M. Boscolo, R. Di Nardo, P. Raimondi, Novel proposal for a low emittance muon beam using positron beam on target (2016). arXiv: 1509.04454, doi:10.1016/j.nima.2015.10.097.

[5] G. Cesarini, M. Antonelli, F. Anulli, M. Bauce, M. E. Biagini, O. R. Blanco-García, M. Boscolo, F. Casaburo, G. Cavoto, A. Ciarma, F. Collamati, C. Daout, R. Li Voti, A. Variola, Theoretical modeling for the thermal stability of solid targets in a positron-driven muon collider, International Journal of Thermophysics 42 (12) (2021) 163. doi:10.1007/ s10765-021-02913-x.

URL https://doi.org/10.1007/s10765-021-02913-x

[6] N. Palmer, et al., Proceedings of the 1996 DPF/DPB Summer Study on High-Energy Physics, in: Stanford Linear Accelerator Center, Menlo Park, CA , 1997.

[7] M. Thomson, Modern Particle Physics, Cambridge University Press, 2013. doi:10.1017/CB09781139525367.

[8] Y. M. Bystritskiy, E. A. Kuraev, G. V. Fedotovich, F. V. Ignatov, Cross sections of muon and charged pion pair production in electron-positron annihilation near the threshold, Phys. Rev. D 72 (2005) 114019. doi: 10.1103/PhysRevD.72.114019.

URL https://link.aps.org/doi/10.1103/PhysRevD.72.114019

[9] N. Amapane, et al., Study of muon pair production from positron annihilation at threshold energy, JINST 15 (01) (2020) P01036. arXiv: 1909.13716, doi:10.1088/1748-0221/15/01/P01036.

[10] F. Iacoangeli, F. Addesa, L. Bandiera, E. Bagli, G. Cavoto., An instrumented absorber detector for hadronic interaction measurements : (The 
Smart Absorber of Crysbeam project), in: 2018 IEEE Nuclear Science Symposium and Medical Imaging Conference, 2018, p. 8824636. doi: 10.1109/NSSMIC. 2018.8824636 .

[11] CAEN Tools for Discovery, https://www.caen.it/products/v1720/.

[12] CAEN Tools for Discovery, https://www.caen.it/products/v1730/.

[13] The Phase-2 Upgrade of the CMS DAQ Interim Technical Design Report, Tech. rep., CERN, Geneva (Sep 2017).

URL https://cds.cern.ch/record/2283193

[14] G. Brianti, et al., SPS North Experimental Area 2013 General Layout, CERN/LAB II/EA/Note (73-4) (1973).

[15] N. Charitonidis, Private Communication (2018).

[16] F. Mandl and G. Shaw, Quantum Field Theory, John Wiley \& Sons, 1984.

[17] C. M. Carloni Calame, An improved parton shower algorithm in qed, Physics Letters B 520 (1-2) (2001) 16-24. doi:10.1016/s0370-2693(01) 01108-x.

URL http://dx.doi.org/10.1016/S0370-2693(01)01108-X

[18] Geant4 Collaboration, https://geant4.web.cern.ch/node/1.

[19] S. Agostinelli, et al., GEANT4: A simulation toolkit, Nucl. Instrum. Meth. A506 (2003) 250-303. doi:10.1016/S0168-9002(03)01368-8.

[20] S. J. Brodsky, R. F. Lebed, Production of the Smallest QED Atom: True Muonium (mu+ mu-), Phys. Rev. Lett. 102 (2009) 213401. arXiv:0904. 2225, doi:10.1103/PhysRevLett.102.213401. 